\title{
Perrault Aux Prises Avec la Fontaine: Imitation, Compétition et Correction Dans Les Fables de Faërne (1699)
}

\author{
Sander Becker
}

Published online: 5 May 2011

(C) The Author(s) 2011. This article is published with open access at Springerlink.com

\begin{abstract}
Known especially for his fairy tales, Charles Perrault is also the author of the Fables de Faërne (1699). In this French translation of the Neo-Latin volume Fabulae Centum (1564), written by the Italian humanist Gabriel Faerno, Perrault had to position himself against his renowned predecessor Jean de La Fontaine, who had been dominating fable literature for decades. Perrault could either imitate his famous example, or evade it, due to anxiety of influence. To illustrate this inner struggle, we systematically compare both authors' fables, concentrating our analysis on versification (metre and rhyme), vocabulary and apostrophe. In our comparison, we constantly verify whether any of the resemblances could be attributable to other French, versified fable books read by both Perrault and La Fontaine. Occasionally, this seems to be the case for the anonymous collection L'Esbatement moral des animaux (1578).
\end{abstract}

Keywords Perrault $\cdot$ La Fontaine $\cdot$ Faërne $\cdot$ Fables $\cdot$ Anxiety of influence

\section{Introduction}

Vers la fin de sa vie, en 1699, Charles Perrault fit paraître ses cent Fables de Faërne, adaptation française du recueil néo-latin Fabulae Centum ${ }^{1}$ de l'humaniste italien Gabriel Faërne (vers 1510-1561). Largement éclipsées par ses propres contes de fée et par les fables de La Fontaine, elles n'ont guère été étudiées jusqu'à

\footnotetext{
1 Nous avons consulté cet ouvrage dans l'édition récente de Marcozzi (2005).
}

S. Becker $(\square)$

Institute for Cultural Disciplines, French Language and Culture,

Leiden University, Witte Singel-complex, Van Wijkplaats 2,

2311 BX Leiden, The Netherlands

e-mail: s.v.becker@umail.leidenuniv.nl 
présent, ${ }^{2}$ à quelques exceptions près. Gabriella Parussa (1988), en comparant la traduction des Fables de Faërne au texte latin, a esquissé quelques correspondances stylistiques entre les fables de Perrault et celles de La Fontaine. Plus récemment, Marc Escola (2005), dans son livre sur Perrault, a noté qu'il serait intéressant d'étudier plus en détail le travail de réécriture auquel celui-ci s'est livré, « moins sur le texte latin de Faërne que sur les deux recueils des Fables de La Fontaine ». C'est dans cette perspective que nous souhaitons analyser ici comment le fabuliste Perrault a réécrit son illustre devancier français, tout en l'imitant ou, au contraire, en le contournant, par anxiety of influence (Bloom, 1973). ${ }^{3}$ Nous nous proposons d'étudier cette réécriture dans le domaine de la versification (mètre et rime), de l'apostrophe et du vocabulaire-analyse comparative vérifiée après une confrontation avec d'autres fabliers versifiés français que Perrault a pu lire, à savoir ceux de Corrozet (1542) et de Haudent (1547), ainsi que L'Esbatement moral des animaux (1578).

\section{Perrault Face à La Fontaine}

Perrault a toujours été un grand admirateur de La Fontaine. Dans Les Hommes illustres (1696-1700), il souligne le «talent merveilleux » du vieux maître de la fable, en louant ses ouvrages pour leur «agrément incomparable ». Il y trouve « une simplicité ingénieuse, une naïveté spirituelle, et une plaisanterie originale qui, n'ayant jamais rien de froid, cause une surprise toujours nouvelle ». Héritage pourtant problématique, comme l'avoue Perrault dans la dédicace de ses Fables de Faërne : il reconnaît avoir longtemps ajourné sa traduction, par crainte de décevoir ses lecteurs, dont les goûts exigent « dans ces sortes d'Ouvrages, de la vivacité \& quelquefois de la plaisanterie qui les surprennent $» 5^{5}$ Les notions de «plaisanterie » et de «surprise » renvoient clairement aux talents de La Fontaine déjà évoqués par Perrault. On pourrait en déduire que Perrault a éprouvé une certaine angoisse devant l'ombre omniprésente du grand fabuliste. On retrouve la même appréhension, de façon encore plus explicite, dans l'Avertissement des Fables de Faërne, où Perrault note qu'il n'oserait comparer ses propres fables à celles de La Fontaine. Il le fait néanmoins, en toute modestie, en s'appuyant sur une métaphore vestimentaire, devenue célèbre : « [L]es nostres [les fables de Faërne et de Perrault] ressemblent à un habit d'une bonne étoffe, bien taillée \& bien cousuë, mais simple \& toute unie; les siennes [de La Fontaine] ont quelque chose de plus, \& il y ajoûte une riche $\&$ fine broderie qui en releve le prix infiniment. ${ }^{6}$ Son

\footnotetext{
$\overline{2}$ À ce sujet, il est intéressant de noter que Jean-Pierre Collinet (2008), dans son excellent article «Perrault et La Fontaine », n'aborde pas les Fables de Perrault. Il se contente seulement d'y faire allusion, en les qualifiant d' « estimable[s], mais sans éclat particulier ».

3 Bloom (1973) analyse le sentiment d'angoisse qu'un poète peut ressentir par rapport à un précurseur admiré : porté à la poésie grâce à l'influence stimulante de ce prédécesseur, il se rend compte qu'il devra se distinguer de lui pour être reconnu à son tour comme un grand poète novateur.

4 Nous soulignons l'usage des deux substantifs par l'ajout d'italique.

5 Perrault (1699), la dédicace à Monsieur l'abbé de Dangeau.

6 Perrault (1699), l'Avertissement.
} 
admiration, à la fois humble et craintive, de La Fontaine nous explique peut-être pourquoi ce n'est qu'après la disparition de ce dernier (en 1695), et sous le couvert de la pédagogie morale, que Perrault a enfin osé publier son recueil de fables en vers. ${ }^{7}$ Le fablier de 1699 accentue ce but pédagogique, non seulement dans la dédicace, mais aussi dans la seule illustration : la gravure du frontispice, accompagnée d'un quatrain de Perrault. Elle montre une femme racontant ses fables devant des enfants. ${ }^{8}$ Le frontispice n'a pas été repris dans les éditions postérieures. $^{9}$

\section{Comparaison Globale Des Deux Fabliers}

$\mathrm{Si}$, malgré la réticence de Perrault, on se hasarde sur le délicat terrain de la comparaison des deux fabliers, on constate d'abord que Perrault, tout comme La Fontaine, a réparti ses fables en plusieurs livres. Son recueil compte cinq volumes, comportant chacun vingt fables, tandis que Faërne, lui, avait numéroté ses fables d'une à cent, sans division. Il s'avère aussi que Perrault a distribué ses fables selon un nouvel ordre, pour lequel il a suivi une édition révisée des Fabulae (1697), imprimée à Paris, deux ans avant la parution de sa traduction. Dans cette réédition latine, on avait choisi de présenter les fables dans un nouvel ordre, adapté à l'enseignement scolaire, c'est-à-dire en mettant les fables les plus courtes et les plus aisées les premières. Cependant, en adoptant cette division en livres, Perrault n'accentue pas seulement ses intentions pédagogiques, mais il renoue aussi implicitement avec la tradition lafontainienne. ${ }^{10}$

En second lieu, on relève que pas moins de trente-cinq des cent Fables de Faërne correspondent à des fables de La Fontaine, parmi lesquelles on compte les plus connues, telles que «La Cigale et la Fourmi » (LF I, 1; P II, 15), « Le Corbeau et le Renard » (LF I, 2; P III, 15) et «Le Chêne et le Roseau » (LF I, 22; P V, 9). ${ }^{11}$

\footnotetext{
${ }^{7}$ Collinet (2008) fait remarquer que les succès de La Fontaine portaient ombrage à Perrault, lequel « rêvait en secret de le supplanter et de prendre sa place quand son prédécesseur serait mort ». En écrivant ses contes, Perrault voulait déjà se démarquer de La Fontaine, devancier dont il se sentait « hanté » et dont «l'obsession l'exaspér[ait]».

${ }^{8}$ La gravure de Jean Mariette porte la légende suivante : « La Fable a des charmes si doux /Pour ces jeunes Enfans dont l'ame est innocente, /Que même sous la forme et des Ours et des Loups, /Son simple recit les enchante. » L'image rappelle le frontispice des Histoires ou Contes du temps passé (1697) de Perrault, où l'on voit une vieille femme-la mère L'Oye — racontant des contes de fées devant un groupe de jeunes enfants. Voir l'article de Smith (2010) sur le rapprochement de ces deux images.

${ }^{9}$ Les Fables de Faërne ont été reproduites dans les Lettres choisies de Messieurs de l'Academie françoise, sur toutes sortes de sujets, Paris 1708 (et dans les rééditions successives de ces Lettres choisies: Paris 1709, Hannover 1709 et Paris 1725), et dans les éditions d'Amsterdam (1718) et de Londres (1741 et 1743). Nous avons consulté toutes ces éditions sauf celle de Paris 1709; aucune de ces éditions ne contient le frontispice de 1699 .

${ }^{10}$ Selon Georges Couton (1962) La Fontaine a réparti ses fables en plusieurs livres pour imiter Phèdre.

${ }^{11}$ Il s' agit des fables suivantes de Perrault : (I, 6), (I, 9), (I, 13), (I, 14), (I, 16), (I, 17), (II, 10), (II, 11), (II, 12), (II, 14), (II, 15), (II, 17), (III, 4), (III, 6), (III, 13), (III, 14), (III, 15), (IV, 3), (IV, 4), (IV, 6), (IV, 7), (IV, 10), (IV, 12), (IV, 16), (IV, 18), (IV, 20), (V, 2), (V, 4), (V, 7), (V, 8), (V, 9), (V, 12), (V, 15), (V, 16) et (V, 19). Pour la citation et la numérotation des fables, nous avons suivi l'édition originale de 1699. Notons que dans certaines éditions postérieures, l'ordre et la numérotation du fablier original de Faërne ont été rétablies.
} 
La dernière, intitulée «La Canne \& l'Olivier » chez Perrault, nous montre que celui-ci n'a en rien cherché à adapter ses titres à ceux de son devancier; il est resté, au contraire, très proche de son modèle néo-latin.

Cela vaut également pour les trames narratives des fables. Prenons « Le Chien, le Coq \& le Renard » $(\mathrm{P} \mathrm{V}, 8)$, intitulée « Le Coq et le Renard » (LF II, 15) chez La Fontaine. Cet apologue met en scène un renard demandant à un coq, installé en toute sécurité au haut d'un arbre, d'en descendre afin de fêter ensemble la paix générale. Dans la version de La Fontaine, le coq matois répond qu'il voit deux lévriers s'approcher, et qu'ils pourront donc bientôt «s'entrebaiser » tous les quatre : ses propos déclenchent la fuite du renard, pressé de sauver sa peau. Chez Perrault, en revanche, le coq ainsi interpellé invite le renard à réveiller le «portier », endormi dans le creux du tronc d'arbre : celui-ci s'avère être un chien féroce qui sort aussitôt de sa tanière et ne tarde pas à « étrangler » le pauvre renard. L'écart entre les deux versions s'explique du fait que Perrault reste très près de sa source néo-latine, alors que La Fontaine suit le modèle du Premier livre des Emblèmes de Guéroult (Lyon 1550). ${ }^{12}$

Même constat pour le reste du recueil : Perrault ne change quasiment rien aux trames de Faërne. ${ }^{13}$ D'ailleurs, le fabuliste le précise lui-même dans son Avertissement, où il qualifie sa traduction de «fort exacte, hors en quelques endroits ou j'ay cru pouvoir m'en dispenser $»{ }^{14}$ Il en résulte que la plupart des «plaisanteries » et des «surprises » qui caractérisent l'écriture de La Fontaine, et que Perrault admirait tant, sont absentes des Fables de Faërne.

\section{Les Rimes}

Pourtant, même si Perrault reste proche du modèle de Faërne, il laisse percer certaines influences textuelles de La Fontaine. Celles-ci se manifestent à plusieurs niveaux, et de façon plus manifeste dans le cas des rimes. Comme le montre le Tableau 1, nous avons relevé trente-trois rimes où au moins un mot lafontainien réapparaît à l'identique chez Perrault. ${ }^{15}$ Apparemment, celui-ci n'a pas rechigné à reprendre des rimes de son prédécesseur. Dans quinze cas (que nous avons indiqués en italique dans le tableau), le deuxième, et même le troisième mot rimant sont également identiques, ce qui, sur un total de trente-cinq fables communes, est considérable. $^{16}$

\footnotetext{
12 Selon l'analyse de Collinet, dans de La Fontaine (1991, p. 1089).

13 Parussa (1988), art. cit., dans son étude de la traduction du texte néo-latin, ne mentionne pas non plus de grands écarts narratifs.

14 Perrault (1699), l'Avertissement.

15 Nous considérons uniquement les rimes dont au moins un mot lafontainien correspond exactement à celui employé par Perrault, et non pas celles ayant seulement le son final en commun; ces dernières sont trop fréquentes et nous semblent dépourvues de signification, car il s'agit souvent de terminaisons finales du genre «-ment » ou «-ie », qui ne renvoient à aucune rime précise de La Fontaine.

16 Dans le tableau, comme dans le reste de l'article, nous utilisons l'orthographe ancienne des fables de Perrault, parce qu'il n'existe pas d'édition moderne. En revanche, pour La Fontaine nous avons préféré l'orthographe moderne, devenue courante.
} 
Tableau 1 Liste de toutes les rimes dont au moins un terme de Perrault correspond exactement à celui employé par La Fontaine

\begin{tabular}{|c|c|c|}
\hline $\mathrm{P} / \mathrm{LF}$ & Perrault & La Fontaine \\
\hline$(\mathrm{I}, 6) /(\mathrm{III}, 11)$ & treille-merveille-pareille* & treille-vermeille \\
\hline \multirow[t]{2}{*}{$(\mathrm{I}, 13) /(\mathrm{III}, 16)$} & bords-corps & corps-bords \\
\hline & riviere-maniere & rivière-arrière \\
\hline \multirow[t]{2}{*}{$(\mathrm{I}, 16) /(\mathrm{VI}, 14)$} & taniere -maniere-poussiere* & poussière-tanière \\
\hline & entre-antre-ventre* & entre-antre \\
\hline (II, 12)/(VI, 7) & inopinément-lourdement-jument* & incessamment-jument \\
\hline (II, 14)/(II, 13) & science-connaissance & Providence-science \\
\hline (III, 13)/(IV, 16) & chaumiere-maniere-mere-colère & fourches-fières - manières-affaire-mère \\
\hline \multirow[t]{2}{*}{ (III, 15)/(I, 2) } & corbeau-oiseau-beau* & corbeau-beau \\
\hline & fromage-branchage-plumage-ramage* & fromage-langage-ramage-plumage \\
\hline \multirow[t]{2}{*}{$(\mathrm{IV}, 3) /(\mathrm{V}, 15)$} & trépas-pas-bras-ingrats* & climats-trépas \\
\hline & mouvement-moment & châtiment-moment \\
\hline \multirow[t]{2}{*}{$(\mathrm{IV}, 4) /(\mathrm{II}, 2)$} & mot-grelot & grelot-sot \\
\hline & projeter-exécuter & délibérer-exécuter \\
\hline$(\mathrm{IV}, 20) /(\mathrm{VI}, 11)$ & seigneur-courroyeur & courroyeur-odeur-seigneur $^{\S}$ \\
\hline \multirow[t]{4}{*}{$(\mathrm{V}, 4) /(\mathrm{II}, 5)$} & $\begin{array}{l}\text { mamelles-ailes, cruelle-éternelle- } \\
\text { telle-elle-aile* }\end{array}$ & nouvelles-ailes \\
\hline & oiseau-moineau-peau* & museau-oiseau \\
\hline & vie-ennemie & étourdie-ennemie-vie-repartie ${ }^{\S}$ \\
\hline & pas-pas & pas-rats-chats ${ }^{\S}$ \\
\hline$(\mathrm{V}, 8) /(\mathrm{II}, 15)$ & peur-trompeur-demeure-l'heure* & peur-trompeur \\
\hline$(\mathrm{V}, 12) /(\mathrm{IV}, 7)$ & usage-voyage-rivage-orage-naufrage* & usage-voyage-équipage-naufrage \\
\hline \multirow[t]{5}{*}{$(\mathrm{V}, 15) /(\mathrm{V}, 1)$} & cognée-destinée & baignée-cognée \\
\hline & encor-d'or & encor-d'or \\
\hline & bien-rien & bien-rien, sien-bien ${ }^{\S}$ \\
\hline & perdue-rendue & entendue-perdue \\
\hline & égale-régale & animal-égal \\
\hline \multirow[t]{7}{*}{$(\mathrm{V}, 19) /(\mathrm{IV}, 22)$} & famille-faucille & famille-faucille ( 2 fois) \\
\hline & demeure-heure & heure-demeure \\
\hline & Aurore-éclore & encore-éclore \\
\hline & mere-affaire & mère-faire \\
\hline & remis-amis et mis-amis* & fils-amis \\
\hline & paix-guérets & jamais-paix \\
\hline & extrême - nous-même & extrême - soi-même \\
\hline
\end{tabular}

En italique, les rimes dont deux ou même trois mots correspondent exactement

L'astérisque * indique les cas où Perrault offre plus de mots rimés que La Fontaine, et, en particulier, un vers où il reprend trois mots rimés de La Fontaine et en remplace un quatrième. Le symbole ${ }^{\S}$ indique les endroits, plus rares, où La Fontaine fait rimer plus de mots que Perrault 
Regardons de plus près un cas exemplaire de ces rimes reprises, à savoir les derniers vers du «Chien, le Coq \& le Renard » $(\mathrm{P}$ V, 8$)$ :

Le Chien au premier coup sortit de sa demeure

Le malheureux Renard pensa mourir de peur

Il fuit, le Chien le prit \& l'étrangla sur l'heure

C'est le vrai droit du jeu de tromper le trompeur.

Dans la fable correspondante, « Le Coq et le Renard » (LF II, 15), La Fontaine finit par:

Adieu, dit le Renard : ma traite est longue à faire.

Nous nous réjouirons du succès de l'affaire

Une autre fois. Le Galand aussitôt

Tire ses grègues, gagne au haut,

Mal content de son stratagème;

Et notre vieux Coq en soi-même

Se mit à rire de sa peur

Car c'est double plaisir de tromper le trompeur.

Malgré les différences de contenu, déjà évoquées, les ressemblances lexicales entre les versions de Perrault et de La Fontaine sautent aux yeux. Tout d'abord, Perrault reprend littéralement l'expression «tromper le trompeur », à caractère hautement proverbial, et absente des fabliers prélafontainiens, du moins chez Corrozet, Haudent ou Baudoin [1631]. En outre, Perrault s'empare de la rime finale « peur-trompeur».

À propos de cette rime dérobée, nous constatons que le fabuliste admirateur ne s'est pas contenté d'un simple larcin : il l'a enrichie, en y ajoutant le couple « demeure-l'heure », la doublant ainsi d'une rime à l'oreille. Une pratique assez courante chez Perrault, qui reprend souvent des rimes en augmentant le nombre de mots rimés : là où La Fontaine livre deux «-eur », par exemple, Perrault s'efforce d'en produire quatre. C'est comme s'il voulait entrer en compétition avec La Fontaine, en se livrant à un procédé que Claude-Gilbert Dubois (1979) a nommé imitation différentielle. ${ }^{17}$ Cette notion renvoie à l'attitude double d'un poète partagé entre un sentiment de reconnaissance envers son modèle et le désir de s'en distinguer. L'imitation différentielle est étroitement liée au concept d'anxiety of influence; elle est la réponse même, concrète et artistique, à l'angoisse du poète. En l'occurrence, la solution choisie par Perrault pour se distinguer de son illustre prédécesseur consiste en une augmentation maniériste du nombre de mots rimés, que nous proposons d'appeler imitation multiplicative.

Ce procédé se reproduit trop souvent pour être le simple fruit du hasard, ce qui nous amène à le considérer comme une véritable forme de rivalité poétique. Citons encore quelques exemples. Dans « Le Singe et le Dauphin » de La Fontaine (LF IV, 7), on trouve « usage-voyage-équipage-naufrage », rime reprise et quelque peu augmentée

${ }^{17}$ Dubois (p. 28) introduit le terme imitation différentielle pour définir l'attitude de l'artiste maniériste face à son modèle. Par extension, ce terme s'applique aussi à d'autres courants que le maniérisme, dès qu'il est question d'un modèle qu'il faut à la fois suivre et contourner. 
par Perrault ( $\mathrm{P} \mathrm{V}, 12)$ sous la forme « usage-voyage-rivage-orage-naufrage ». Pour la fable «Le Lion malade et le Renard»(LF VI, 14), où La Fontaine propose « poussière-tanière » et « entre-antre », Perrault (P I, 16) surenchérit encore en livrant «taniere-maniere-poussiere » et « entre-antre-ventre ». Au total, nous avons compté pas moins de douze cas, indiqués par un astérisque (*) dans le tableau, où Perrault cherche à l'emporter en nombre sur les rimes lafontainiennes. Ces rimes sont réparties sur neuf fables différentes, soit un quart des trente-cinq fables communes aux deux auteurs. À l'inverse, nous n'avons recensé que quatre cas, répartis sur trois fables, où les rimes de La Fontaine sont plus nombreuses que celles de Perrault, ainsi que l'indique le signe $\S$ dans le tableau. En conclusion, il en ressort que les reprises de Perrault ne sont en rien fortuites, mais plutôt la marque d'une volonté auctoriale.

L'exemple le plus frappant de ces emprunts poétiques à La Fontaine est celui du «Corbeau et le Renard », même si ce cas s'avère quelque peu problématique. Au premier abord, la fable présente plusieurs rimes indéniablement tirées de La Fontaine. On y entend bourdonner les échos du maître dès les six premiers vers:

Perrault (P III, 15):

Sur le haut d'un chêne, un Corbeau

Tenoit dans son bec un fromage;

Quel est ce merveilleux Oiseau

Que je voy là sur ce branchage ?

Dit un Renard qu'il est grand, qu'il est beau !

Rien n'approche de son plumage;

La Fontaine (LF I, 2):

Maître Corbeau, sur un arbre perché,

Tenait en son bec un fromage.

Maître Renard, par l'odeur alléché,

Lui tint à peu près ce langage :

Et bonjour, Monsieur du Corbeau,

Que vous êtes joli ! que vous me semblez beau !

Sans mentir, si votre ramage

Se rapporte à votre plumage,

Vous êtes le Phénix des hôtes de ces bois.

Avant de considérer ces rimes dans le détail, prêtons attention à quelques ressemblances frappantes à première vue, mais qui portent la trace de subtiles transformations lorsqu' on y regarde de plus près. Le deuxième vers des deux fables est ainsi quasiment identique, à l'exception de la préposition « en », que Perrault remplace par « dans ». Le premier vers, où les deux fabulistes présentent à peu près les mêmes informations, subit lui aussi une légère modification : Perrault ne positionne plus le corbeau au début du vers, comme le faisait La Fontaine, mais à la fin. Même type d'inversement dans les vers qui suivent : La Fontaine introduit d'abord son renard pour le faire parler ensuite, tandis que Perrault commence par la citation, ne mentionnant le locuteur qu'après. Ce faisant, Perrault évite d'emblée de diriger le lecteur dans le sillon lafontainien. Prises dans leur ensemble, ses adaptations révèlent une certaine anxiété de l'influence : la crainte de passer pour un 
fade imitateur incite Perrault à prendre ses distances, même si les éléments qu'il copie (le mètre, le ton léger et surtout les rimes) révèlent, dans le même temps, toute l'admiration qu'il porte à son prédécesseur.

Mais revenons aux rimes. Là où La Fontaine produit « Corbeau-beau », Perrault le suit et le dépasse en ajoutant « oiseau ». Le lecteur instruit du XVIIe siècle-tout comme le lecteur d'aujourd'hui-, qui connaissait par cœur la fable de La Fontaine, eut sans doute l'impression que Perrault se livrait de nouveau à son jeu de l'imitation multiplicative_-effet probablement recherché par celui-ci. Pourtant, lors d'une comparaison méticuleuse des sources, il s'avère que les trois rimes finissant en «-eau » étaient déjà présentes dans le recueil anonyme L'Esbatement moral des animaux (1578), ${ }^{18}$ longtemps avant l'entrée en scène de La Fontaine et de Perrault. C'est aussi plus ou moins le cas pour les rimes finissant en «-age »: La Fontaine semble avoir emprunté trois mots rimés finissant en «-age » de L'Esbatement moral (« fromage-ramage-plumage ») ${ }^{19}$ et avoir remplacé le quatrième, « hommage », par « langage ». Perrault a lui aussi repris le premier triplet, mais en l'enrichissant cette fois du mot «branchage ». S'il semble évident que La Fontaine a puisé sa rime dans L'Esbatement moral, la question reste posée concernant Perrault : s'est-il inspiré directement de son prédécesseur? ou bien a-t-il lu le même recueil que lui ? En ce cas, les deux fabulistes pourraient s'en être inspirés indépendamment l'un de l'autre, tout en y apportant leur propre variation. ${ }^{20}$ Mais on pourrait également s'interroger sur les motivations de Perrault et concevoir qu'il a précisément repris ces rimes pour nuire à La Fontaine, selon le principe de daemonization. Cette notion, formulée par Bloom dans sa théorie de l'anxiety of influence, définit l'une des six stratégies dont dispose un poète pour se distinguer d'un précurseur. ${ }^{21}$ Concrètement, appliqué au cas de Perrault, cela induit que le fabuliste aurait cherché à diminuer l'originalité présumée de La Fontaine, en

${ }^{18}$ L'Esbat[e]ment moral des animaux (1578):

Ce Regnard, qui voyoit au bec de ce Corbeau

Une bien bonne part de quelque gras formage,

Ie voy bien maintenant, dict il, que ton plumage,

Contre le bruict commun, est excellamment beau.

La raison requiert bien, amy, que tout oiseau,

$\mathrm{Si}$, dy-ie, à ta blancheur respondoit ton ramage,

Pour ton merite grand, te vienne faire hommage,

Et qu'on t'eslise Roy sur eux tout de nouveau.

L'Esbatement moral a été plagié par Philippe Desprez, qui en a repris cent fables dans Le Théatre des Animaux (Paris, 1595; rééditions en 1613, 1620 et 1644). Pourtant, il est improbable que La Fontaine et Perrault se soient basés sur ce recueil pirate, car quelques-unes des fables qu'ils ont imitées figurent uniquement dans L'Esbatement moral, et sont absentes du Théâtre des Animaux.

19 Smith (2005) fait également cette observation.

${ }^{20}$ Chez Haudent, on retrouve aussi « corbeau-beau » et «fourmage-hommage-dommage »; quant à Corrozet, il avait déjà fait rimer «corbeau-beau» et «plumage-avantage ». Mais chez ces deux fabulistes, 'oiseau' et 'ramage' manquent dans la rime, ce qui fait que L'Esbatement moral constitue la source la plus complète et la plus probable de La Fontaine et de Perrault.

21 Selon Bloom, op. cit., un poète dispose de six stratégies différentes pour se distancier d'un précurseur. Il les appelle clinamen (déviation correctrice de l'œuvre inspiratrice), tessera (complétion et antithèse), kenosis (répétition et discontinuité), daemonization (recours à la source d'inspiration du précurseur, en diminuant ainsi l'originalité présumée de sa contribution), askesis (purgation) et apophrades (retour à l'imitation, mais d'une façon maîtrisée et consciente). 
mettant en avant le fait que celui-ci a copié de nombreux éléments poétiques de l'Esbatement moral.

La présence de l'Esbatement moral dans les Fables de Faërne est encore plus évidente dans «Le Renard \& les Raisins » (P I, 6). Perrault y produit la rime «treille-merveille-pareille », que l'on est porté à interpréter de nouveau comme une imitation variée et enrichie de la paire «treille-vermeille », forgée par La Fontaine (LF III, 11). ${ }^{22}$ Pourtant, L'Esbatement moral nous propose déjà « treille-merveille-appareille-conseille », et une lecture comparative des deux fabulistes prouve que la version de Perrault reste la plus proche de celle du recueil anonyme : la seule nouveauté de Perrault consiste à avoir supprimé les deux premières lettres d' « appareille », tandis que La Fontaine avait choisi de changer un mot entier, remplaçant « merveille» par « vermeille». Perrault a également repris d'autres termes caractéristiques de L'Esbatement moral, comme « peine » et «verjus ». Dans deux autres fables, il semble encore une fois imiter cet ouvrage. ${ }^{23}$ Ces correspondances multiples renforcent l'idée que Perrault a bien consulté ce recueil, tout comme l'avait fait La Fontaine avant lui. ${ }^{24}$

\section{Le Vocabulaire}

Si les fables de La Fontaine ont pu influencer le travail de versification de Perrault, elles ont aussi constitué pour lui une large source d'inspiration lexicale. Parussa a ainsi relevé un art de camper les animaux chez La Fontaine que Perrault a cherché à imiter. $^{25}$ Elle mentionne notamment trois épithètes dans les Fables de Faërne qui évoquent l'ombre de son précurseur : le renard est « compère » (P I, 16; LF I, 18), la fourmi est «ménagère » (P II, 15; LF IV, 3), et le loup de Perrault est un « grand mangeur de chair cruë » $(\mathrm{P}$ II, 6), proche en cela du renard lafontainien qualifié de « grand croqueur de poulets, grand preneur de lapins » (LF V, 5).

Nous avons déjà constaté que Perrault avait recyclé presque un vers entier de La Fontaine, dans « Le Corbeau \& le Renard» (P III, 15). Pareillement dans «La Fourmi \& la Cigale » (P II, 15), où il reprend le dernier vers « Eh bien ! dansez maintenant », pour le modifier en « Eh bien dansez donc aujourd'hui », transformation à laquelle nous reviendrons pour aborder la question du mètre.

En outre, dans «La Canne \& l'Olivier» $(\mathrm{P} \mathrm{V}, 9)$, Perrault puise chez son devancier l'expression « moindre vent » et le terme « aquilon » (LF I, 22). Plus

\footnotetext{
${ }^{22}$ Parussa l'interprète ainsi, art. cit., p. 111.

23 Il s'agit de la fable «Le Lion, l'Âne \& le Renard» $\mathrm{P}$ V, 3), où l'on trouve «partagesage-dommage » chez Perrault, à côté de « sage-présage » dans L'Esbatement moral, ainsi que de la fable « Le Satyre \& l'Homme » $(\mathrm{P} I \mathrm{~V}, 6)$, où Perrault, proposant « doigts-quelquefois-droit-froid », s'est visiblement inspiré des rimes « bois-doigts-vois-fois » de L'Esbatement moral.

24 Nous n'avons pas pu retrouver les autres rimes concurrentes de Perrault et de La Fontaine dans les fabliers de Corrozet et de Haudent, qui, après L'Esbatement moral, constituaient les seules sources françaises en vers. Cependant, Haudent (II, 37) présente déjà la rime « peine-vaine » et la rime un peu maladroite « poin[c]t-point » à la fin de sa fable, toutes deux présentes dans « Le Renard \& les Raisins » de Perrault. Ce dernier a donc peut-être également consulté Haudent.

25 Parussa, art. cit., pp. 108-9.
} 
remarquable, Perrault s'empare aussi du mot «arbuste », que La Fontaine avait utilisé pour désigner le roseau. Cette qualification peut surprendre, car le mot « arbuste » est habituellement réservé aux végétaux ligneux et ramifiés, et non à ces plantes aquatiques, aux tiges droites, qui bordent les étangs. La Fontaine l'a sans doute employé parce qu'il en avait besoin pour rimer avec « injuste ». Or, la fable de Faërne, où la canne et l'olivier se querellent et s'injurient, offre à Perrault une excellente occasion d'apporter une correction. Perrault fait ainsi dire à l'olivier, s'adressant à la canne : « Toi qui n'est [sic] pas même un arbuste ». Cette subtile reprise lexicale nous semble s'adresser, de façon détournée et malicieuse, à La Fontaine, d'autant que le mot « arbuste » ne figure pas dans l'original de Faërne et que Perrault aurait donc pu aisément s'en passer. Ce mécanisme, coulé dans le moule théorique de l'anxiety of influence, relèverait de la notion de tessera: le poète retient un terme de son précurseur, tout en lui donnant une tournure nouvelle.

\section{Les Mètres}

L'influence textuelle de La Fontaine se manifeste aussi au niveau des mètres. Dans chaque fable, Perrault emploie des vers d'une longueur variable : octosyllabes, décasyllabes et alexandrins alternent, procédé copié directement du maître du vers varié. Dans leur choix du mètre, les deux fabulistes se laissent également guider par des considérations stylistiques comparables. Par exemple, ils réservent souvent les alexandrins aux effets de grandeur ou d'emphase. Ainsi, dans « Le Corbeau et le Renard », les fabulistes les intercalent afin d'exprimer surtout les fausses flatteries et l'apothéose de la morale, frôlant ainsi un registre élevé. ${ }^{26}$

Perrault (III, 15):

Si vous charmez l'oüye aussi bien que la veuë, [12]

Je vous tiens le plus beau des habitans de l'air, [12]

Sans même en excepter l'oiseau de Jupiter. [12]

$[\ldots]$

Loüer en face est une lâche ruse, [10]

Et pour s'y laisser prendre, il faut être bien buse. [12]

Pourtant, des divergences frappantes demeurent entre les deux fabulistes. Perrault, tout moderne qu'il est, se montre plus conservateur que La Fontaine, et s'abstient d'utiliser une versification trop libre à son goût. Les vers hepta-, tétra-, tri- ou même dissyllabiques, que l'on croise souvent chez La Fontaine, sont complètement bannis des Fables de Faërne. ${ }^{27}$ Perrault a même corrigé, d'une manière voyante, un vers lafontainien de sept syllabes, déjà mentionné ci-dessus : le

\footnotetext{
${ }^{26}$ La Fontaine (I, 2) : «Que vous êtes joli ! que vous me semblez beau ! [12 syllabes]/[...]/Vous êtes le Phénix des hôtes de ces bois. [12]/[...]/Cette leçon vaut bien un fromage sans doute. [12] ».

${ }^{27}$ Nous avons compté un seul vers comportant neuf syllabes, entouré d'octosyllabes. Il s'agit du vers « Dans la Salle Royale il entra », dans la fable «Le Lion, le Loup \& le Renard » (P V, 16). C'est une erreur, car la mise en page n'inclut pas de retraits à gauche: les octosyllabes restent alignés sur ce vers de neuf syllabes.
} 
célèbre « Eh bien ! dansez maintenant », énoncé par la fourmi peu prêteuse (LF I, 1), s'enrichit, sous la plume de Perrault (P II, 15), d'une huitième syllabe, et devient «Eh bien dansez donc aujourd'hui ». Apparemment, pour Perrault, le « donc » ajoute juste ce qu'il faut pour compléter le vers et le rendre régulier. Soulignons, par ailleurs, que la variante « aujourd'hui » est assez recherchée, car le texte latin propose «nunc », ce qui correspond exactement à « maintenant ». En changeant ce mot, Perrault a sans doute voulu s'écarter encore quelque peu de La Fontaine, étant donné qu'il avait déjà entièrement recyclé la structure de son vers.

Mais Perrault ne se limite pas à désapprouver ouvertement les vers déviants. Car si La Fontaine se plaît parfois à présenter des fables ne comportant justement qu'un seul type de vers, ${ }^{28}$ Perrault préfère éviter cette uniformité jugée, probablement, trop monotone. Chez ce dernier, aucune fable ne présente une versification entièrement régulière; l'alternance est devenue une règle stricte. En ce sens, le fablier de Perrault est plus unifié ou équilibré que celui de La Fontaine.

Une dernière différence réside dans le fait que La Fontaine tend davantage à l'expérimentation formelle : il ose quitter une certaine routine stylistique. En composant, par exemple, «Le Lion malade et le Renard » (LF VI, 14) presque entièrement en octosyllabes_-22 au total—, il choisit d'y inclure deux alexandrins qui se détachent de ce bloc rectangulaire. Et c'est justement dans ces deux vers que le renard découvre le drame affreux qui se joue : le lion, feignant une maladie, dévore tous les animaux qui lui rendent visite, car, d'après les empreintes sur le sol, aucun ne revient sur ses pas pour se sauver de l'antre royal...

Les pas empreints sur la poussière [8]

Par ceux qui s'en vont faire au malade leur cour, [12]

Tous, sans exception, regardent sa tanière; [12]

Pas un ne marque de retour. [8]

Ces deux alexandrins rompent la monotonie rythmique, suscitant l'attention du lecteur et renforçant le choc du contenu. Chez Perrault, la fable correspondante (« Le Lion \& le Renard », P I, 16) offre une alternance de vers beaucoup moins abrupte, et par conséquent moins surprenante. Pourtant, dans une autre fable, Perrault, incidemment, semble se rappeler le procédé audacieux de La Fontaine, et en prendre exemple. Dans « L'Asne \& le Renard » (P II, 10), où un âne déguisé en lion se trahit par sa voix ridicule, une suite de huit alexandrins est rompue par un seul décasyllabe, et s'achève sur un octosyllabe. Ce dernier vers, qui frappe et par sa longueur réduite, et par son placement à la fin, permet ainsi de bien envoyer la morale:

Du superbe Ignorant, dés qu'il rompt le silence, [12]

On voit l'orgueil \& l'ignorance. [8]

Toutefois, chez Perrault ces expérimentations restent rares. Il est évident qu'il ne cherche pas à produire autant d'effets de surprise que La Fontaine, ni de façon aussi radicale. En conclusion, on peut avancer que Perrault a copié, voire relevé au niveau

\footnotetext{
${ }^{28}$ Pour rompre le procédé de l'alternance continuelle, La Fontaine a choisi parfois de composer des fables comportant uniquement des octosyllabes (par exemple IV, 1 et 7), des alexandrins (XII, 25), ou même des heptasyllabes (I, 9 et IV, 6).
} 
d'une norme absolue, les caractéristiques les plus constantes de la versification variée de La Fontaine, tout en rejetant les tendances les plus « déviantes ».

En dehors de la rime et du mètre, bien d'autres aspects poétiques des deux fabulistes se ressemblent. Perrault ne copie pas littéralement La Fontaine, mais il se sert des mêmes outils pour agrémenter ses vers. Sans entrer dans le détail, relevons, en particulier, le recours à l'allitération et à la répétition. ${ }^{29}$

\section{Vouvoyer, Tutoyer}

Pour rendre ses fables plus vivantes, Perrault, tout comme La Fontaine, fait souvent parler ses animaux en style direct. Parfois il peut se contenter de traduire les dialogues de Faërne, mais lorsque ce dernier se limite au discours indirect, Perrault doit les inventer lui-même. Parussa a déjà signalé qu'il fait généralement parler ses personnages «comme des hommes du XVII ${ }^{\mathrm{e}}$ siècle », c'est-à-dire qu'ils se vouvoient et affectent un langage raffiné. ${ }^{30}$

Le vouvoiement dans les fables ne semblait pas être encore très en vogue avant La Fontaine. Prenons l'exemple du « Corbeau et le Renard». Chez La Fontaine et Perrault, le renard vouvoie sa victime pour rendre ses flatteries plus efficaces. Mais ce n'était pas le cas chez leurs devanciers : dans l'Esbatement moral, ainsi que chez Corrozet, Haudent et Baudoin, le renard de cette même fable se sert de la deuxième personne du singulier. Ces auteurs restaient en cela plus proches de la tradition latine, également présente chez Faërne, où les animaux se tutoient. Même R. D. F. [Raphaël Trichet du Fresne], dans son fablier de 1659, suit encore le tutoiement ancien.

On est tenté d'en déduire l'hypothèse selon laquelle le vouvoiement a été introduit au XVII ${ }^{\mathrm{e}}$ siècle, lorsque la fable fut adaptée en français, et que La Fontaine en est l'instigateur. Pourtant, ce serait peut-être lui faire trop d'honneur, car déjà chez Corrozet, on rencontre parfois quelques «vous » isolés. C'est le cas dans «Du Loup et de l'Agneau » (no. 2), où le loup brutal tutoie l'agneau innocent, qui, à son tour, vouvoie le loup, en le traitant de « seigneur ». Cette opposition, qui souligne les rapports hiérarchiques entre les deux animaux, réapparaît telle quelle chez La Fontaine (LF I, 10). Toujours est-il que les «vous » restent rares dans les fables prélafontainiennes. On peut donc néanmoins soutenir que La Fontaine a été le premier fabuliste à exploiter pleinement le vouvoiement et le tutoiement. Chez lui, le

\footnotetext{
29 Ainsi, dans les premiers vers de « L'Astrologue » (P II, 14), Perrault évoque bien le pas lent et rêveur de l'astrologue au moyen d'une allitération en « 1 »: « Un Astrologue allant la nuit,/Les yeux levés au ciel », faisant ainsi écho aux « pas pesants » du bûcheron lafontainien (I, 16). À propos de la répétition, citons « La Femme noyée \& son Mary » (P I, 13), où le mari en question cherche sa femme noyée en amont d'une rivière, et non pas en aval, car « cette vieille obstinée,/a fait \& fait encor toute chose à rebours ». La répétition de «fait», soulignant que le caractère têtu de la femme ne changera jamais, rend à merveille l'état plaintif du mari. La Fontaine utilise encore le même procédé pour accentuer la taille élancée de son héron (VII, 4): « Un jour sur ses longs pieds allait je ne sais où/Le Héron au long bec emmanché d'un long cou ».

30 Parussa, art. cit., p. 109.
} 
choix entre «tu $»$ ou « vous » a toujours un sens, tandis que chez ses devanciers, le « tu » restait surtout la norme.

Or, c'est exactement cet emploi varié et raisonné du vouvoiement que Perrault a copié de La Fontaine. Lui aussi s'en sert pour esquisser des rapports hiérarchiques, comme dans « Le Lion, le Loup \& le Renard » $(\mathrm{P} V, 16)$, où un renard se voit trahi devant le roi-lion par un loup calomniateur. Dans cette situation menaçante, le renard, à l'instar de tout sujet en présence de son monarque, vouvoie le lion; par contre, il tutoie le traître loup, après s'être vengé de lui en le faisant écorcher vivant. Le tutoiement augmente encore l'humiliation de l'adversaire.

En imitant cette forme de politesse animalière, Perrault va très loin. Il ne reprend pas seulement le principe en soi du vouvoiement, mais aussi la façon plaisante de passer du « vous» au «tu » que La Fontaine a introduite dans « Le Renard, le Singe et les animaux » (LF VI, 6). Dans cette fable, le renard essaie d'abord de flatter le roi-singe : il lui promet un trésor et lui adresse des courtoisies, lui donnant du «Sire » et du «Votre Majesté ». Mais dès que le singe est tombé dans le piège, le renard lafontainien change radicalement de ton:

Prétendrais-tu nous gouverner encor,

$\mathrm{Ne}$ sachant pas te conduire toi-même ?

Même coup de théâtre dans la fable équivalente de Perrault (P IV, 16). Au départ, le renard y traite le singe de "Prince », en le vouvoyant, pour frapper ensuite d'autant plus fort avec son « tu » railleur. La seule autre fable correspondante en vers français se trouve dans le recueil de Haudent (II, 21). Celui-ci emploie le tutoiement dès le début, et c'est un «tu » dépourvu de charge moqueuse, puisque ce pronom se manifeste dans à peu près toutes ses fables où se développe un dialogue en discours direct. On en déduit que Perrault a en effet tiré son modèle dramaturgique de La Fontaine.

Mais si, pour l'apostrophe, Perrault suit l'approche raisonnée de La Fontaine, au final, leur choix d'adresse n'en est pas pour autant identique, loin de là. Par exemple, dans « Le Coq et le Renard » (LF II, 15), les deux animaux lafontainiens se traitent de « Frère » et d' "Ami », en se tutoyant. Pour tromper sa victime, le renard s'en prend ici d'une manière tout à fait différente de celle qu'il avait tentée précédemment avec le corbeau : les flatteries courtoises ont cédé leur place à l'amitié feinte, allant bien sûr de pair avec le tutoiement. Perrault (P V, 8), à l'inverse, choisit de faire parler son renard au style indirect, d'un ton plus courtois qu'amical. Par conséquent, il ne serait pas logique que son coq, qui prend ensuite la parole, tutoie le renard. Aussi ce vieux et sage gallinacé use-t-il d'un ton formel et distancié:

Je n'ay pas de plus grand desir,

Que de vous donner du plaisir,

Mais si vous voulez que je sorte,

Il faut éveiller le Portier,

Afin qu'il nous ouvre la Porte,

Oserois-je vous en prier ?

Par ailleurs, chez La Fontaine et Perrault, les mêmes animaux sont en général tutoyés-les ânes (trop bêtes) et les loups (détestés)—, ou vouvoyés—les lions 
(royaux). Mais les deux fabulistes adaptent la recette à leur gré. Dans « L'Ane portant des reliques » (LF V, 14), La Fontaine met ainsi en scène un homme vouvoyant un âne, parce que ce dernier se prend pour un dieu. Pure ironie ici, qui consiste à s'adresser au plus stupide des animaux avec une politesse démesurée:

Maître Baudet, ôtez-vous de l'esprit

Une vanité si folle.

Ce n'est pas à vous, c'est l'Idole

A qui cet honneur se rend

Cependant, le tutoiement irrespectueux de l'animal présomptueux que nous propose Perrault dans sa fable correspondante (P I, 14) se défend aussi, et la leçon de morale n'en est pas moins caustique:

Alors à grands coups de baston

On lui rabaissa le courage.

Apprens, bourique, lui dit-on,

Qu'au Dieu, non pas à toi, se rend tout cet hommage.

Penchons-nous enfin sur une célèbre fable, où Perrault se distancie clairement de l'exemple fourni par La Fontaine. Il s'agit de « La Mort et le Malheureux » (LF I, 15), où, dès le début, on est frappé par le fait que le miséreux de La Fontaine tutoie la mort. On s'attendrait en effet à un peu plus de distance à l'égard de cette apparition tant redoutée. Ce fut sans doute l'avis de Perrault (P II, 17), qui semble, dans sa version de la fable, corriger le choix de La Fontaine ${ }^{31}$ : l'humble et pauvre vieillard y vouvoie «le spectre épouventable [sic] », qui, de son côté, tutoie sa victime.

\section{Conclusion}

L'approche comparative présentée ici nous a révélé maintes ressemblances entre les fables en vers de Perrault et celles de La Fontaine, notamment au niveau des rimes. Perrault n'a pas seulement repris de nombreux mots rimés par son prédécesseur, il semble également avoir voulu entrer en compétition avec lui. D'ailleurs, lorsqu'il utilise parfois des rimes de L'Esbatement moral, il reprend les mêmes dont La Fontaine s'est sans doute inspiré. Du reste, nous avons mis en lumière quelques termes employés dans les Fables de Faërne qui semblent directement tirés de La Fontaine. Perrault n'a pas non plus hésité à corriger son précurseur français dès qu'il jugeait le choix de ses mots un peu trop malheureux. De même pour les mètres trop

\footnotetext{
31 La fable de La Fontaine a également été critiquée par Boileau. Celui-ci en a proposé sa propre version, sans changer le tutoiement, qui, sans doute, ne lui semblait pas problématique. Voici ses derniers vers:

La mort vient à la fin. Que veux-tu ? cria-t-elle.

Qui, moi ? dit-il alors, prompt à se corriger.

Que tu m'aides à me charger.

Ce tutoiement évoque peut-être aussi une certaine familiarité que les vivants ressentent face à la mort. Il pourrait s'agir également d'une allusion sous-entendue à l'expresssion «tutoyer la mort », qui s'emploie quand on la frôle de près.
} 
irréguliers, ou au contraire trop réguliers à son goût. Ce procédé de correction dans le domaine du mètre nous a amené à conclure que Perrault a cherché à formaliser la pratique lafontainienne à alterner des vers de longueur différente. Il s'est également livré à des jeux de langage qui rappellent les procédés d'allitération et de répétition employés par son prédécesseur français. À cela s'ajoute l'imitation de l'emploi raisonné du vouvoiement et du tutoiement, y compris dans la façon de passer avec humour du «vous » au «tu ». Malgré l'anxiety of influence dont Perrault semblait souffrir, il a donc trouvé une façon de s'émanciper de son modèle, en adoptant une attitude à la fois admirative, compétitive et critique.

Cependant, il faut se rendre compte que les ressemblances relevées ici ne résultent pas nécessairement d'une imitation de La Fontaine, ou de l'un des autres fabulistes français mentionnés dans cet article. Des poètes étrangers-notamment Giovan Mario Verdizotti, qui s'est inspiré de Faërne ${ }^{32}$ - peuvent aussi avoir laissé des traces. De plus, le hasard n'est pas à exclure. Dans son Avertissement des Fables de Faërne, Perrault lui-même évoque déjà "l'impossibilité qu'il y a que deux personnes qui travaillent sur un mesme sujet ne se rencontrent pas quelque fois dans les mesmes pensées ou dans les mesmes expressions ». Même s'il commente ici les analogies accidentelles entre Faërne et le fabuliste ancien Phèdre, il est séduisant d'interpréter ce passage comme un reflet de son propre rapport à La Fontaine. Comme si Perrault s'assurait d'avance contre la critique d'avoir copié sur son illustre devancier. Ainsi, confronté aux parentés discutées ci-dessus, Perrault aurait sans doute été parmi les premiers à relativiser leur importance.

Remerciements Je tiens à remercier M. Smith pour ses encouragements et sa direction du travail, tout comme Aurore Evain pour sa lecture critique de l'article.

Open Access This article is distributed under the terms of the Creative Commons Attribution Noncommercial License which permits any noncommercial use, distribution, and reproduction in any medium, provided the original author(s) and source are credited.

\section{Ouvrages cités}

Anonyme. (1578). L'Esbat[e]ment moral des animaux. Anvers: Philippe Galle.

Baudoin, J. (1631). dans l'édition de 1649. Les Fables d'Ésope Phrygien. Paris: Jean du Bray.

Bloom, H. (1973). The anxiety of influence: A theory of poetry. New York: Oxford University Press.

Collinet, J.-P. (2008). Perrault et La Fontaine. Romanic Review, 99(3/4), 191-209.

Corradi, F. (2008). Giovan Mario Verdizotti et le renouveau de la fable ésopique en vers dans l'Italie du $\mathrm{XVI}^{\mathrm{e}}$ siècle. Le Fablier, 19, 37-46.

Corrozet, G. (1542). dans l'édition de 1596. Les Fables d'Esope. Genève: Jean de Tournes.

Couton, G. (1962). Introduction aux Fables choisies mises en vers. Paris: éditions Garnier Frères.

de La Fontaine, J. (1991). Euvres complètes, vol. I, Fables, contes et nouvelles, édition établie par JeanPierre Collinet. Paris: Gallimard.

Dubois, C.-G. (1979). Le Maniérisme. Paris: Presses universitaires de France.

Escola, M. (2005). Contes de Charles Perrault (pp. 178-179). Paris: Gallimard.

Faerno, G. (1564). Fabulae Centum, ex antiquis auctoribus delectae et a Gabriele Faerno Cremonensi carminibus explicatae.

\footnotetext{
32 Pour l'influence de Verdizotti sur La Fontaine, voir Corradi (2008).
} 
Faerno, G. (1697). Fabulae ex Aesopo, aliisque priscis autoribus. Paris: Apud Viduam Claudii Thiboust. Bibliothèque universitaire de l'Arsenal de Toulouse, cote Res Mn 9088.

Haudent, G. (1547). dans l'édition de 1877. Trois cent soixante et six Apologues d'Ésope traduicts en rithme françoise. Rouen: Jehan le Prest.

Marcozzi, L. (2005). Le Favole. Rome: Salerno.

Parussa, G. (1988). Deux essais de traduction: Les fables de Francesco Filelfo traduites par Jean Baudoin et celles de Gabriele Faerno traduites par Charles Perrault. Reinardus, I, 104-112.

Perrault, C. (1699). Traduction des fables de Faërne. Paris: Coignard. Cote BNF: YC-9756.

Perrault, C. (1696-1700). dans l'édition de 2003. Les Hommes illustres qui ont paru en France pendant ce siècle (pp. 212-215). Tübingen: Culpin, Gunter Narr.

R. D. F. [Raphaël Trichet du Fresne]. (1659). Figures tirées des Fables d'Ésope et d'autres et expliquées par R.D.F. Paris: Claude Cramoisy.

Smith, P. J. (2005). La Fontaine et Ogilby, Chauveau et Hollar: Imitations poétiques et picturales. Le Fablier, 16, 19-25.

Smith, P. J. (2010). Fables ésopiques et contes de fées. L'imitation différentielle dans les frontispices. Relief revue électronique de littérature française, 4(2), 27-51. 